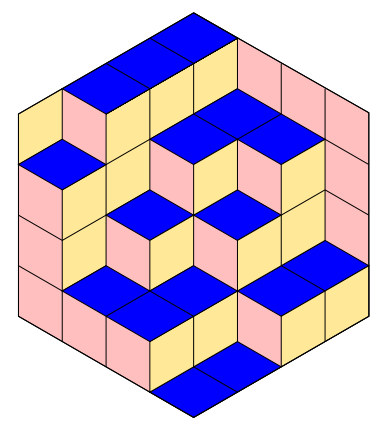

ALGEBRAIC COMBINATORICS

Karola Mészáros \& Linus Setiabrata

Lorentzian polynomials from polytope projections

Volume 4, issue 4 (2021), p. 723-739.

<http://alco.centre-mersenne.org/item/ALCO_2021__4_4_723_0>

(C) The journal and the authors, 2021.

Some rights reserved.

(c) BY This article is licensed under the

Creative Commons ATtribution 4.0 InTERnational License.

http://creativecommons.org/licenses/by/4.0/

Access to articles published by the journal Algebraic Combinatorics on the website http://alco.centre-mersenne.org/ implies agreement with the Terms of Use (http://alco.centre-mersenne.org/legal/).

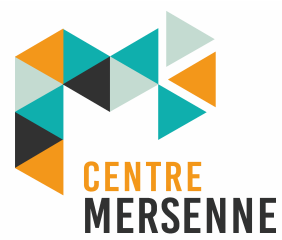

Algebraic Combinatorics is member of the Centre Mersenne for Open Scientific Publishing www.centre-mersenne.org 


\title{
Lorentzian polynomials from polytope projections
}

\author{
Karola Mészáros \& Linus Setiabrata
}

\begin{abstract}
Lorentzian polynomials, recently introduced by Brändén and Huh, generalize the notion of log-concavity of sequences to homogeneous polynomials whose supports are integer points of generalized permutahedra. Brändén and Huh show that normalizations of integer point transforms of generalized permutahedra are Lorentzian. Moreover, normalizations of certain projections of integer point transforms of generalized permutahedra with zero-one vertices are also Lorentzian. Taking this polytopal perspective further, we show that normalizations of certain projections of integer point transforms of flow polytopes are Lorentzian.
\end{abstract}

\section{INTRODUCTION}

The classical notion of log-concavity of sequences is often either a very easy or a notoriously difficult property to prove. A sequence $a_{0}, a_{1}, \ldots, a_{n}$ is said to be log-concave if $a_{i}^{2} \geqslant a_{i-1} a_{i+1}$ for $i \in[n-1]$. In groundbreaking recent work Brändén and Huh [3] introduced Lorentzian polynomials (see Section 2.1 for definition), which generalize the notion of log-concavity. Just one of their theory's many consequences are the celebrated Alexandrov-Fenchel inequalities on mixed volumes of Minkowski sums of polytopes; these inequalities follow from the Lorentzian property of the volume polynomial [3, Theorem 9.1]. In [1], Lorentzian polynomials were used to give a generalization of Postnikov's formula for the volume of a generalized permutahedron, a beautiful polytope studied extensively in [9]. In [4], Lorentzian polynomials were also used to prove a generalization of Mason's conjecture on the $f$-vectors of independent subsets of matroids.

Our motivation for the present paper is simple: we want to understand Lorentzian polynomials polytopally.

Recall that for a polytope $P \subset \mathbb{R}^{n}$, the integer point transform of $P$ is defined as

$$
\sigma_{P}\left(x_{1}, \ldots, x_{n}\right)=\sum_{\mathbf{p} \in P \cap \mathbb{Z}^{n}} \mathbf{x}^{\mathbf{p}}, \quad \text { where } \quad \mathbf{x}^{\mathbf{p}}=\prod_{i=1}^{n} x_{i}^{p_{i}} .
$$

Define the normalization operator $N$ on $\mathbb{R}\left[x_{1}, \ldots, x_{n}\right]$ by

$$
N\left(\mathbf{x}^{\alpha}\right)=\frac{\mathbf{x}^{\alpha}}{\alpha !}
$$

Manuscript received 20th September 2020, revised 19th April 2021, accepted 21st April 2021.

KEYWORDS. Lorenztian polynomial, flow polytope, integer point transforms.

ACKnowledgements. Karola Mészáros received support from NSF Grant DMS-1501059, CAREER NSF Grant DMS-1847284 and a von Neumann Fellowship funded by the Friends of the Institute for Advanced Study. 
where for a vector $\alpha=\left(\alpha_{1}, \ldots, \alpha_{n}\right)$ of nonnegative integers we write $\alpha$ ! to mean $\prod_{i=1}^{n} \alpha_{i} !$

By definition, the support of a Lorentzian polynomial forms the integer points of a generalized permutahedron. Brändén and Huh show that the normalization of the integer point transform of a generalized permutahedron is always Lorentzian $[3$, Theorem 7.1(4),(7)]. When these generalized permutahedra have vertices in $\{0,1\}^{n}$, certain projections of their integer point transforms are also Lorentzian, by [3, Theorem 2.10] and [3, Corollary 6.7]. In joint work with Huh, Matherne and St. Dizier, the first author showed that the normalization of certain projections of the integer point transforms of Gelfand-Tsetlin polytopes are Lorentzian [5, Theorem 1]. The question lurking in the background of the present work is:

QUESTION 1.1. Which polytope/projection pairs give rise to normalized projected integer point transforms that are Lorentzian?

The current paper adds a natural class of polytope/projection pairs yielding Lorentzian polynomials: flow polytopes, with projection onto a coordinate subspace.

The flow polytope $\mathcal{F}_{G}(\mathbf{a})$ associated to a loopless graph $G$ on the vertex set $[n+1]$ with edges directed from smaller to larger vertices and to the netflow vector $\mathbf{a}=$ $\left(a_{1}, \ldots, a_{n+1}\right) \in \mathbb{Z}^{n+1}$ is:

$$
\mathcal{F}_{G}(\mathbf{a})=\left\{f \in \mathbb{R}_{\geqslant 0}^{E(G)}: M_{G} f=\mathbf{a}\right\}
$$

where $M_{G}$ is the incidence matrix of $G$; that is, the columns of $M_{G}$ are the vectors $e_{i}-e_{j}$ for $(i, j) \in E(G), i<j$, where $e_{i}$ is the $i$-th standard basis vector in $\mathbb{R}^{n+1}$. The points $f \in \mathcal{F}_{G}(\mathbf{a})$ are called (a-)flows (on $G$ ).

Observe that the number of integer points in $\mathcal{F}_{G}(\mathbf{a})$ is the number of ways to write a as a nonnegative integral combination of the vectors $e_{i}-e_{j}$ for edges $(i, j)$ in $G$. This number is the Kostant partition function $K_{G}(\mathbf{a})$.

We define two natural projections $\varphi$ and $\psi$ of $\mathcal{F}_{G}(\mathbf{a})$ onto generalized permutahedra in Propositions 3.4 and 3.6 in Section 3. The projections $\varphi$ and $\psi$ induce projections on the integer point transform $\sigma_{\mathcal{F}_{G}(\mathbf{a})}(\mathbf{x})$ of $\mathcal{F}_{G}(\mathbf{a})$, acting on monomials via $\mathbf{x}^{f} \mapsto \mathbf{x}^{\varphi(f)}$ and $\mathbf{x}^{f} \mapsto \mathbf{x}^{\psi(f)}$. The resulting projected polynomials are denoted

$$
\sigma_{G(\mathbf{a})}^{\varphi}(\mathbf{x}) \stackrel{\text { def }}{=} \sum_{\mathbf{p} \in \mathcal{F}_{G}(\mathbf{a}) \cap \mathbb{Z}|E(G)|} \mathbf{x}^{\varphi(\mathbf{p})},
$$

and

$$
\sigma_{G(\mathbf{a})}^{\psi}(\mathbf{x}) \stackrel{\text { def }}{=} \sum_{\mathbf{p} \in \mathcal{F}_{G}(\mathbf{a}) \cap \mathbb{Z}|E(G)|} \mathbf{x}^{\psi(\mathbf{p})} .
$$

While the normalization of the integer point transform of $\mathcal{F}_{G}(\mathbf{a})$ is not Lorentzian in general, we prove that the normalizations of its projections $\sigma_{G(\mathbf{a})}^{\varphi}$ and $\sigma_{G(\mathbf{a})}^{\psi}$ are always Lorentzian:

THEOREM 5.1. Let $G$ be a loopless directed graph on the vertex set $[n+1]$ with a unique sink, and let $\mathbf{a}=\left(a_{1}, \ldots, a_{n+1}\right) \in \mathbb{Z}_{\geqslant 0}^{n} \times \mathbb{Z}_{\leqslant 0}$. The polynomials $N\left(\sigma_{G(\mathbf{a})}^{\varphi}\right)$ and $N\left(\sigma_{G(\mathbf{a})}^{\psi}\right)$ are Lorentzian.

Theorem 5.1 implies that the Kostant partition function is log-concave along root directions (Corollary 5.2). We remark that log-concavity of the Kostant partition function along root directions is also a corollary of volume polynomials (of flow polytopes) being Lorentzian (Theorem 2.6). 
ROADMAP OF THE PAPER. Section 2 contains the necessary background on Lorentzian polynomials, generalized permutahedra and flow polytopes. Section 3 introduces the projections $\varphi$ and $\psi$ of $\mathcal{F}_{G}(\mathbf{a})$ onto generalized permutahedra that we are interested in, while Section 4 studies their fibers. Section 5 establishes our main result, Theorem 5.1 . Section 6 prods Question 1.1.

\section{BACKGROUND}

In this section we give background on the main players of the paper: Lorentzian polynomials, generalized permutahedra and flow polytopes.

2.1. LORENTZIAN POLYNOMIALS AND GENERALIZED PERMUTAHEDRA. Let $\mathbb{N}=$ $\{0,1,2, \ldots\}$, and denote by $e_{i}$ the $i$-th standard basis vector of $\mathbb{N}^{n}$. A subset $J \subseteq \mathbb{N}^{n}$ is called M-convex if for any index $i$ and any $\alpha, \beta \in J$ whose $i$-th coordinates satisfy $\alpha_{i}>\beta_{i}$, there is an index $j$ satisfying

$$
\alpha_{j}<\beta_{j}, \quad \alpha-e_{i}+e_{j} \in J, \text { and } \beta-e_{j}+e_{i} \in J .
$$

The convex hull of an M-convex set is a polytope also called a generalized permutahedron. A special class of generalized permutahedra consist of Minkowski sums of scaled coordinate simplices: for a subset $S \subseteq[n]$, the coordinate simplex $\Delta_{S} \subseteq \mathbb{R}^{n}$ is the convex hull of the coordinate basis vectors $\left\{e_{i}\right\}_{i \in S}$. Minkowski sums of scaled coordinate simplices are called y-generalized permutahedra.

Let $\mathrm{H}_{n}^{d}$ be the space of degree $d$ homogeneous polynomials with real coefficients in the $n$ variables $x_{1}, \ldots, x_{n}$. For $f \in \mathrm{H}_{n}^{d}$, we write $\operatorname{supp}(f) \subseteq \mathbb{N}^{n}$ for the support of $f$. For $f \in \mathrm{H}_{n}^{d}$, denote by $\frac{\partial}{\partial x_{i}} f$ the partial derivative of $f$ relative to $x_{i}$. The Hessian of a homogeneous quadratic polynomial $f \in \mathrm{H}_{n}^{2}$ is the symmetric $n \times n$ matrix $H=$ $\left(H_{i j}\right)_{i, j \in[n]}$ defined by $H_{i j}=\partial_{i} \partial_{j} f$. The set $\mathrm{L}_{n}^{d}$ of Lorentzian polynomials with degree $d$ in $n$ variables is defined as follows. Set $\mathrm{L}_{n}^{1} \subseteq \mathrm{H}_{n}^{1}$ to be the set of all linear polynomials with nonnegative coefficients. Let $\mathrm{L}_{n}^{2} \subseteq \mathrm{H}_{n}^{2}$ be the subset of quadratic polynomials with nonnegative coefficients whose Hessians have at most one positive eigenvalue and which have M-convex support. For $d>2$, define $\mathrm{L}_{n}^{d} \subseteq \mathrm{H}_{n}^{d}$ recursively by

$$
\mathrm{L}_{n}^{d}=\left\{f \in \mathrm{M}_{n}^{d}: \frac{\partial}{\partial x_{i}} f \in \mathrm{L}_{n}^{d-1} \text { for all } i\right\} .
$$

where $\mathrm{M}_{n}^{d} \subseteq \mathrm{H}_{n}^{d}$ is the set of polynomials with nonnegative coefficients whose supports are M-convex.

Since $f \in \mathrm{M}_{n}^{d}$ implies $\frac{\partial}{\partial x_{i}} f \in \mathrm{M}_{n}^{d-1}$, we have

$$
\mathrm{L}_{n}^{d}=\left\{f \in \mathrm{M}_{n}^{d}: \frac{\partial}{\partial x_{i_{1}}} \frac{\partial}{\partial x_{i_{2}}} \cdots \frac{\partial}{\partial x_{i_{d-2}}} f \in \mathrm{L}_{n}^{2} \text { for all } i_{1}, i_{2}, \ldots, i_{d-2} \in[n]\right\} .
$$

Recall the normalization operator $N$ on $\mathbb{R}\left[x_{1}, \ldots, x_{n}\right]$ :

$$
N\left(\mathbf{x}^{\alpha}\right)=\frac{\mathbf{x}^{\alpha}}{\alpha !}
$$

where for a vector $\alpha=\left(\alpha_{1}, \ldots, \alpha_{n}\right)$ of nonnegative integers we write $\alpha$ ! to mean $\prod_{i=1}^{n} \alpha_{i} !$

For a quadratic polynomial

$$
f(\mathbf{x})=\sum_{1 \leqslant i \leqslant j \leqslant n} c_{i j} x_{i} x_{j} \in \mathrm{M}_{n}^{2}
$$

observe that the $i j$-th entry of the Hessian of $N(f)$, namely the quantity $\partial_{i} \partial_{j} N(f)$, is the coefficient $c_{i j}$ of $x_{i} x_{j}$ in $f$. Thus, asking whether $N(f)$ is Lorentzian, equivalently whether the Hessian of $N(f)$ has at most one positive eigenvalue, can be phrased 
purely in terms of the coefficients of $f$. For arbitrary polynomials $f \in \mathrm{M}_{n}^{d}$ we use the following lemma:

Lemma 2.1. The linear operator $N^{-1} \frac{\partial}{\partial x_{i}} N$ acts on polynomials by

$$
\left(N^{-1} \frac{\partial}{\partial x_{i}} N\right): \sum_{\alpha} c_{\alpha} \mathbf{x}^{\alpha} \mapsto \sum_{\alpha: \alpha_{i} \geqslant 1} c_{\alpha} \mathbf{x}^{\alpha-e_{i}}
$$

We arrive at the following criterion for Lorentzian polynomials.

LEMma 2.2. Let $f$ be a homogeneous polynomial of degree $d \geqslant 2$, and suppose

$$
f(\mathbf{x})=\sum_{\alpha} c_{\alpha} \mathbf{x}^{\alpha} \in \mathrm{M}_{n}^{d} .
$$

For each $\mathbf{d}=\left(d_{1}, \ldots, d_{n}\right)$ with $d_{1}+\cdots+d_{n}=d-2$ and $d_{i} \in \mathbb{Z}_{\geqslant 0}$ for $i \in[n]$, define the $n \times n$ matrix

$$
H_{\mathbf{d}}=\left(H_{i j ; \mathbf{d}}\right)_{i, j \in[n]} ; \quad H_{i j ; \mathbf{d}}=c_{\mathbf{d}+e_{i}+e_{j}}
$$

consisting of coefficients of $f$. Then $N(f) \in \mathrm{L}_{n}^{d}$ if and only if $H_{\mathbf{d}}$ has at most one positive eigenvalue for each $\mathbf{d}$.

Proof. Note that normalization and differentiation preserve M-convexity of the support of a polynomial. By Lemma 2.1, we obtain

$$
N^{-1} \partial_{\mathbf{d}} N(f)=\sum_{\alpha: \alpha \geqslant \mathbf{d}} c_{\alpha} \mathbf{x}^{\alpha-\mathbf{d}} \in \mathrm{M}_{n}^{2}
$$

Because the Hessian of $N\left(N^{-1} \partial_{\mathbf{d}} N(f)\right)=\partial_{\mathbf{d}} N(f)$ is $H_{\mathbf{d}}$, by definition $N(f)$ is Lorentzian if and only if $H_{\mathbf{d}}$ has at most one positive eigenvalue for each $\mathbf{d}$.

The coefficients of Lorentzian polynomials satisfy a log-concavity inequality as in Proposition 2.3 below. It is in this sense that Lorentzian polynomials generalize the notion of log-concavity.

Proposition 2.3 ([3, Proposition 9.4]). If $f(\mathbf{x})=\sum_{\alpha} c_{\alpha} \mathbf{x}^{\alpha}$ is a homogeneous polynomial on $n$ variables so that $N(f)$ is Lorentzian, then for any $\alpha \in \mathbb{N}^{n}$ and any $i, j \in[n]$ the inequality

holds.

$$
c_{\alpha}^{2} \geqslant c_{\alpha+e_{i}-e_{j}} c_{\alpha-e_{i}+e_{j}}
$$

This proposition can be seen as a consequence of Cauchy's Interlacing Theorem. We recall below a special case of Cauchy's Interlacing Theorem, which we will use later.

Proposition 2.4 (Cauchy's Interlacing Theorem, [8, Theorem 10.1.1]). Let $A$ be a symmetric $n \times n$ matrix, and let $S \subseteq[n]$, and $m=|S|$. Let $B=A_{S}$ be the $m \times m$ principal submatrix of $A$ given by $B=\left(a_{i j}\right)_{i, j \in S}$. Let $\alpha_{1} \leqslant \cdots \leqslant \alpha_{n}$ be the eigenvalues of $A$ and let $\beta_{1} \leqslant \cdots \leqslant \beta_{m}$ be the eigenvalues of $B$. Then for every $j \in[m]$,

$$
\alpha_{j} \leqslant \beta_{j} \leqslant \alpha_{n-m+j} .
$$

In other words, the $j$ th smallest eigenvalue of $A$ is at most the $j$ th smallest eigenvalue of $B$, and the $j$ th largest eigenvalue of $A$ is at least the $j$ th largest eigenvalue of $B$.

We recall two important theorems about Lorentzian polynomials here:

TheOREM 2.5 ([3, Theorem 2.10]). If $f \in \mathrm{L}_{n}^{d}$ is a Lorentzian polynomial in $n$ variables, and $A$ is an $n \times m$ matrix with nonnegative entries, then $f(A \mathbf{v}) \in \mathrm{L}_{m}^{d}$ is a Lorentzian polynomial in the $m$ variables $\mathbf{v}=\left(v_{1}, \ldots, v_{m}\right)$. 
TheOREM 2.6 ([3, Theorem 9.1]). Let $K=\left(K_{1}, \ldots, K_{n}\right)$ be convex bodies in $\mathbb{R}^{d}$. The volume polynomial

$$
\left(w_{1}, \ldots, w_{n}\right) \mapsto \operatorname{vol}\left(w_{1} K_{1}+\cdots+w_{n} K_{n}\right)
$$

is a Lorentzian polynomial.

2.2. Flow polytopes. Recall the definition of flow polytopes in (3). We record several properties of them here which we will be using in later sections.

LEMMA 2.7 ([10]). For any graph $G$ on the vertex set $[n+1]$, the vertices of the flow polytope $\mathcal{F}_{G}\left(e_{1}-e_{n+1}\right)$ are unit flows with support equal to $\mathrm{p}$, where $\mathrm{p}$ is an increasing path from vertex 1 to vertex $n+1$.

Proposition 2.8 ([2, Section 3.4]). For nonnegative integers $a_{1}, \ldots, a_{n}$ and $G$ a graph on the vertex set $[n+1]$ we have that

$$
\mathcal{F}_{G}(\mathbf{a})=a_{1} \mathcal{F}_{G}\left(e_{1}-e_{n+1}\right)+a_{2} \mathcal{F}_{G}\left(e_{2}-e_{n+1}\right)+\cdots+a_{n} \mathcal{F}_{G}\left(e_{n}-e_{n+1}\right) .
$$

The following explicit formula for the volume of a flow polytope, combined with Theorem 2.6 and Lemma 2.2, give many matrices with at most one positive eigenvalue. The proof of Theorem 5.1 will then proceed by applying Lemma 2.2 in the opposite direction.

Theorem 2.9 (Baldoni-Vergne volume formula, [2, Theorem 38]). Let G be a directed graph on the vertex set $[n+1]$ with a unique sink, so that edges are oriented from a smaller vertex to a larger vertex. Let $\mathbf{x}=\left(x_{1}, \ldots, x_{n},-\sum_{i=1}^{n} x_{i}\right), x_{i} \in \mathbb{Z}_{\geqslant 0}$. Then

$$
\operatorname{vol} \mathcal{F}_{G}(\mathbf{x})=\sum_{\mathbf{j}} K_{G}\left(j_{1}-\text { out }_{1}, \ldots, j_{n}-\text { out }_{n}, 0\right) \frac{\mathbf{x}^{\mathbf{j}}}{\mathbf{j} !},
$$

for out ${ }_{i}=\operatorname{outdeg}_{i}-1$, where outdeg ${ }_{i}$ denotes the outdegree of vertex $i$ in $G$. The sum is over weak compositions $\mathbf{j}=\left(j_{1}, \ldots, j_{n}\right)$ of $|E(G)|-n$ that dominate $\left(\right.$ out $_{1}, \ldots$, out $\left._{n}\right)$, that is, for every $i \in[n]$ we have

$$
j_{1}+\cdots+j_{i} \geqslant \text { out }_{1}+\cdots+\text { out }_{i} .
$$

In the above $\mathbf{x}^{\mathbf{j}}=\prod_{i=1}^{n} x_{i}^{j_{i}}$ and $\mathbf{j} !=\prod_{i=1}^{n} j_{i} !$.

REMARK 2.10. Suppose $G$ is a directed graph on the vertex set $[n+1]$ with a unique sink. Then the Kostant partition function $K_{G}(\mathbf{v})$ is nonzero only when $\mathbf{v}$ dominates 0. Thus, Baldoni-Vergne's formula (Theorem 2.9) could be stated as

$$
\operatorname{vol} \mathcal{F}_{G}(\mathbf{x})=\sum_{\mathbf{j}} K_{G}\left(j_{1}-\text { out }_{1}, \ldots, j_{n}-\text { out }_{n}, 0\right) \frac{\mathbf{x}^{\mathbf{j}}}{\mathbf{j} !},
$$

where the sum runs over weak compositions $\mathbf{j}=\left(j_{1}, \ldots, j_{n}\right)$ of $|E(G)|-n$.

\section{Projections of Flow polytopes onto Generalized PERMUTAHEDRA}

In this section, we define the projections $\varphi: \mathcal{F}_{G}(\mathbf{a}) \rightarrow \mathcal{P}(G ; \mathbf{a})$ and $\psi: \mathcal{F}_{G}(\mathbf{a}) \rightarrow$ $\mathcal{Q}(G ; \mathbf{a})$, where $\mathcal{P}(G ; \mathbf{a})$ and $\mathcal{Q}(G ; \mathbf{a})$ are $y$-generalized permutahedra (see Propositions 3.4 and 3.6). We study their fibers in Section 4, leading us to explicit expressions for the polynomials $\sigma_{G(\mathbf{a})}^{\varphi}$ and $\sigma_{G(\mathbf{a})}^{\psi}$; see Corollary 4.3. In Section 5 we use these expressions to prove Theorem 5.1. 
Notational Conventions for Sections 3, 4 And 5. Unless specified otherwise, $G$ denotes a loopless directed graph on the vertex set $[n+1]$ with a unique sink. Every edge of $G$ is oriented from its smaller vertex to its larger vertex. All flow polytopes $\mathcal{F}_{G}(\mathbf{a})$ have netflow vector $\mathbf{a} \in \mathbb{Z}_{\geqslant 0}^{n} \times \mathbb{Z}_{\leqslant 0}$. For a finite set $S$, we denote by $\mathbb{R}^{S}$ the real vector space consisting of $\mathbb{R}$-linear combinations of elements in $S$; in particular, for sets $S \subseteq T$, the vector space $\mathbb{R}^{S}$ is a coordinate subspace of $\mathbb{R}^{T}$. We write $\mathbb{R}^{n}$ to denote $\mathbb{R}^{[n]}$.

Definition 3.1. For $i, j \in V(G)=[n+1]$, we denote by $M(i, j) \in \mathbb{N}_{\geqslant 0}$ the number of edges from $i$ to $j$, and by $\{(i, j ; k)\}_{k \in[M(i, j)]} \subseteq E(G)$ the set of edges of $G$ connecting $i$ to $j$.

Definition 3.2 (see Example 3.3). We denote by $S_{G}$ the set of all edges incident to the sink, that is,

$$
S_{G} \stackrel{\text { def }}{=}\{e \in E(G): e=(i, n+1 ; k) \text { for some } i \in[n], k \in[M(i, n+1)]\} .
$$

For $i \in[n]$, let $S_{G, i} \subseteq S_{G}$ be the set of edges incident to $n+1$ which can be reached from vertex $i$, that is, if $\bar{G}$ denotes the transitive closure of $G$, then

$$
S_{G, i} \stackrel{\text { def }}{=}\left\{e \in S_{G}: e=(j, n+1 ; k) \text { and }(i, j) \in E(\bar{G})\right\} .
$$

Denote by $T_{G}$ the set of all vertices incident to the sink, that is,

$$
T_{G} \stackrel{\text { def }}{=}\{i \in V(G): M(i, n+1) \geqslant 1\} .
$$

For $i \in[n]$, let $T_{G, i} \subseteq T_{G}$ be the set of vertices adjacent to $n+1$ which can be reached from vertex $i$, that is,

$$
T_{G, i} \stackrel{\text { def }}{=}\left\{j \in T_{G}:(i, j) \in E(\bar{G})\right\} .
$$

ExAmple 3.3. Let $G$ be as in Figure 1. The set $S_{G} \subseteq E(G)$ consists of the blue edges, while $S_{G, 2}$ consists of the four blue edges emanating from vertices 2 and 4 . If $G^{\prime}$ denotes the graph obtained from $G$ by removing the edge $(2,3 ; 1) \in E(G)$, then $S_{G^{\prime}, 2}$ would only consist of the two blue edges emanating from vertex 2 .

The set $T_{G} \subseteq V(G)=[5]$ is equal to $\{1,2,4\}$, and $T_{G, 3}=\{4\}$.

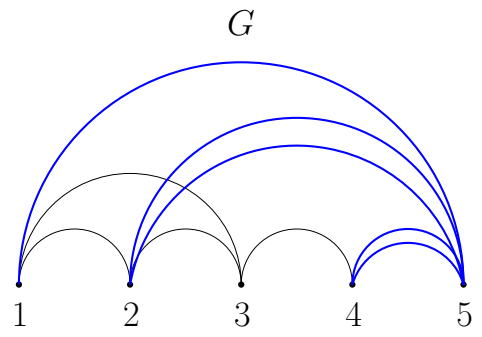

Figure 1. A graph $G$ satisfying the conventions of this section, with edge orientations suppressed.

Proposition 3.4. Let $\varphi$ denote the map sending a flow in $\mathcal{F}_{G}(\mathbf{a})$ to the coordinates corresponding to edges in $S_{G}$. Then $\varphi$ is a projection

$$
\varphi: \mathcal{F}_{G}(\mathbf{a}) \rightarrow \mathcal{P}(G ; \mathbf{a}),
$$

where $\mathcal{P}(G ; \mathbf{a}) \subseteq \mathbb{R}^{S_{G}}$ is the y-generalized permutahedron defined by

$$
\mathcal{P}(G ; \mathbf{a}) \stackrel{\text { def }}{=} \sum_{i \in[n]} a_{i} \Delta_{S_{G, i}} .
$$


Proof. Proposition 2.8 asserts that

$$
\mathcal{F}_{G}(\mathbf{a})=\sum_{i \in[n]} a_{i} \mathcal{F}_{G}\left(e_{i}-e_{n+1}\right) .
$$

Because linear maps factor through Minkowski sums, we obtain

$$
\varphi\left(\mathcal{F}_{G}(\mathbf{a})\right)=\sum_{i \in[n]} a_{i} \varphi\left(\mathcal{F}_{G}\left(e_{i}-e_{n+1}\right)\right) .
$$

Observe that $\varphi\left(\mathcal{F}_{G}\left(e_{i}-e_{n+1}\right)\right)=\Delta_{S_{G, i}}$, because their vertex sets coincide: Lemma 2.7 asserts that the vertices of $\mathcal{F}_{G}\left(e_{i}-e_{n+1}\right)$ are unit flows on paths $\mathrm{p}$ from $i$ to $n+1$; under $\varphi$, the vertex of $\mathcal{F}_{G}\left(e_{i}-e_{n+1}\right)$ corresponding to $\mathrm{p}$ is mapped to the vertex of $\Delta_{S_{G, i}}$ corresponding to the (unique) edge in $\mathrm{p}$ that is incident to $n+1$. The claim $\varphi\left(\mathcal{F}_{G}(\mathbf{a})\right)=\mathcal{P}(G ; \mathbf{a})$ follows.

We note that a special case of Proposition 3.4 was considered in [7, Section 4].

Definition 3.5. For $i \in[n]$, let $I_{i}$ denote the set of $M(i, n+1)$ coordinates in $\mathbb{R}^{E(G)}$ corresponding to an edge connecting $i$ to $n+1$. For a flow $x \in \mathcal{F}_{G}(\mathbf{a})$, define the escaping flow vector ef $(x)=\left(\operatorname{ef}(x)_{1}, \ldots\right.$, ef $\left.(x)_{n}\right) \in \mathbb{R}^{n}$ coordinatewise by

$$
\text { ef }(x)_{i} \stackrel{\text { def }}{=} \sum_{j \in I_{i}} x_{j} .
$$

For $x \in \mathcal{F}_{G}(\mathbf{a})$, and $\varphi$ as in Proposition 3.4 , define

$$
\text { ef }(\varphi(x)) \stackrel{\text { def }}{=} \text { ef }(x) \text {. }
$$

Note that ef $(x)$ depends only on coordinates of $x \in \mathcal{F}_{G}(\mathbf{a}) \subseteq \mathbb{R}^{E(G)}$ indexed by an edge $e \in S_{G}$. Hence ef $(\varphi(x)) \stackrel{\text { def }}{=}$ ef $(x)$ is well defined since $\varphi$ leaves the coordinates of $x$ corresponding to edges in $S_{G}$ unchanged.

Note also that if $i \notin T_{G}$, or equivalently that if $I_{i}=\varnothing$, then ef $(x)_{i}=0$. Thus, we may regard ef $(x)$ as a vector in $\mathbb{R}^{T_{G}}$ (however, it will be useful to regard them as elements of $\mathbb{R}^{n}$ whose coordinates indexed by $[n] \backslash T_{G}$ are zero).

Proposition 3.6. Let $\psi$ denote the map sending $x \mapsto \operatorname{ef}(x)$. Then $\psi$ is a projection

$$
\psi: \mathcal{F}_{G}(\mathbf{a}) \rightarrow \mathcal{Q}(G ; \mathbf{a})
$$

where $\mathcal{Q}(G ; \mathbf{a}) \subseteq \mathbb{R}^{T_{G}}$ is the y-generalized permutahedron defined by

$$
\mathcal{Q}(G ; \mathbf{a}) \stackrel{\text { def }}{=} \sum_{i \in[n]} a_{i} \Delta_{T_{G, i}} .
$$

The map $\psi$ factors through $\varphi$, that is, the following diagram commutes:

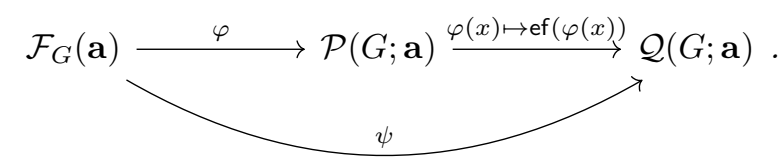

Proof. As in the proof of Proposition 3.4, it will suffice to show $\psi\left(\mathcal{F}_{G}\left(e_{i}-e_{n+1}\right)\right)=$ $\Delta_{T_{G, i}}$. Lemma 2.7 asserts that that the vertices of $\mathcal{F}_{G}\left(e_{i}-e_{n+1}\right)$ are unit flows on paths $\mathrm{p}$ from $i$ to $n+1$; under $\psi$, the vertex of $\mathcal{F}_{G}\left(e_{i}-e_{n+1}\right)$ corresponding to $\mathrm{p}$ is mapped to the vertex of $\Delta_{T_{G, i}}$ corresponding to the (unique) vertex $t$ of $G$ for which $\mathrm{p}$ contains an edge from $t$ to $n+1$.

The statement that the diagram commutes boils down to the fact that ef $(\varphi(x)) \stackrel{\text { def }}{=}$ ef $(x)$ is well defined, as discussed after Definition 3.5. 


\section{THE FIBERS OF $\varphi$ AND $\psi$}

In order to study $\sigma_{G(\mathbf{a})}^{\varphi}$ and $\sigma_{G(\mathbf{a})}^{\psi}$ as defined in (4) and (5), we rewrite them as in equations (8) and (9) below; these equations follows from Propositions 3.4 and 3.6. Equations (8) and (9) make it evident that in order to explicitly compute the coefficients of the monomials appearing in $\sigma_{G(\mathbf{a})}^{\varphi}$ and $\sigma_{G(\mathbf{a})}^{\psi}$ (Corollary 4.3) we need to compute the fibers of $\varphi$ and $\psi$, which is what we accomplish in Theorem 4.1 and Corollary 4.2, respectively.

For brevity of notation, we index the coordinates of a point $\mathbf{x} \in \mathbb{R}^{S_{G}}$ with $(i ; k)$, which is shorthand for the edge $(i, n+1 ; k) \in S_{G}$. We define the polynomials

$$
\sigma_{G(\mathbf{a})}^{\varphi}\left(x_{i ; k}\right)=\sum_{\mathbf{p} \in \mathcal{P}(G ; \mathbf{a}) \cap \mathbb{Z}^{S_{G}}}\left|\varphi^{-1}(\mathbf{p}) \cap \mathbb{Z}^{E(G)}\right| \mathbf{x}^{\mathbf{p}},
$$

and

$$
\sigma_{G(\mathbf{a})}^{\psi}\left(x_{i}\right)=\sum_{\mathbf{p} \in \mathcal{Q}(G ; \mathbf{a}) \cap \mathbb{Z}^{T}}\left|\psi^{-1}(\mathbf{p}) \cap \mathbb{Z}^{E(G)}\right| \mathbf{x}^{\mathbf{p}} .
$$

THEOREM 4.1. Given a point $\mathbf{p} \in \mathcal{P}(G ; \mathbf{a})$, the preimage $S_{\mathbf{p}} \stackrel{\text { def }}{=} \varphi^{-1}(\mathbf{p})$ is a translation of the flow polytope $\mathcal{F}_{G}\left(a_{1}-\operatorname{ef}(p)_{1}, \ldots, a_{n}-\operatorname{ef}(p)_{n}, 0\right)$. For $\mathbf{p} \in \mathbb{Z}^{S_{G}}$, the polytope $S_{\mathbf{p}}$ is integrally equivalent to $\mathcal{F}_{G}\left(a_{1}-\operatorname{ef}(p)_{1}, \ldots, a_{n}-\operatorname{ef}(p)_{n}, 0\right)$.

We emphasize that $\mathbf{a}=\left(a_{1}, \ldots, a_{n},-\sum_{i=1}^{n} a_{i}\right)$ with $a_{i} \geqslant 0$, and that for any $\mathbf{p} \in \mathcal{P}(G ; \mathbf{a})$ we have

$$
\sum_{i=1}^{n} \operatorname{ef}(p)_{i}=\sum_{i=1}^{n} \sum_{j \in I_{i}} p_{j}=\sum_{j \in S_{G}} p_{j}=\sum_{i=1}^{n} a_{i},
$$

with the second equality by the fact that $\bigsqcup_{i} I_{i}=S_{G}$ and the last equality by the definition of $\mathcal{P}(G ; \mathbf{a})$.

Proof of Theorem 4.1. Let $\varphi^{\perp}: \mathbb{R}^{E(G)} \rightarrow \mathbb{R}^{E(G)}$ denote the projection sending components corresponding to edges in $S_{G}$ to zero. Note that $\varphi$ and $\varphi^{\perp}$ project $\mathbb{R}^{E(G)}$ to orthogonal complements, so $\varphi^{\perp}$ is necessarily an injection from $S_{\mathbf{p}}$ onto its image (since points in $S_{\mathbf{p}}$ are all mapped to $\mathbf{p}$ by $\varphi$ ). To clean up notation, we write $z_{i}=a_{i}-\operatorname{ef}(p)_{i}$.

Restricting an a-flow in $S_{\mathbf{p}}$ onto the edges in $\left.G\right|_{[n]}$ gives a (nonnegative) flow with netflow precisely $a_{i}-\operatorname{ef}(p)_{i}$ on vertex $i$. Hence, $\varphi^{\perp}$ is a map $S_{\mathbf{p}} \hookrightarrow \mathcal{F}_{G}\left(z_{1}, \ldots, z_{n}, 0\right)$; furthermore, the inverse $\mathcal{F}_{G}\left(z_{1}, \ldots, z_{n}, 0\right) \rightarrow S_{x}$ is translation by

$$
\tilde{\mathbf{p}}=\left(\tilde{p}_{e}\right)_{e \in E(G)} \in \mathbb{R}^{E(G)} ; \quad \tilde{p}_{e} \stackrel{\text { def }}{=} \begin{cases}p_{e} & \text { if } e \in S_{G} \\ 0 & \text { otherwise }\end{cases}
$$

Hence, $S_{\mathbf{p}}$ is equal to $\mathcal{F}_{G}\left(z_{1}, \ldots, z_{n}, 0\right)$ up to translation by $\tilde{\mathbf{p}}$. Furthermore, if $\mathbf{p} \in$ $\mathbb{Z}^{S_{G}}$, then translation by $\tilde{\mathbf{p}} \in \mathbb{Z}^{E(G)}$ is an integral equivalence $S_{\mathbf{p}} \equiv \mathcal{F}_{G}\left(z_{1}, \ldots, z_{n}, 0\right)$.

Corollary 4.2. Given a point $\mathbf{p} \in \mathcal{Q}(G ; \mathbf{a})$, the preimage $T_{\mathbf{p}} \stackrel{\text { def }}{=} \psi^{-1}(\mathbf{p})$ is equal to $\mathcal{F}_{G}\left(a_{1}-p_{1}, \ldots, a_{n}-p_{n}, 0\right) \times \prod_{i \in T_{G}} p_{i} \Delta_{I_{i}}$.

Proof. Observe that $\varphi^{\perp}\left(T_{\mathbf{p}}\right)=\mathcal{F}_{G}\left(a_{1}-p_{1}, \ldots, a_{n}-p_{n}, 0\right)$, since an a-flow in $T_{\mathbf{p}}$ restricted onto just the edges in $\left.G\right|_{[n]}$ gives a flow with netflow precisely $a_{i}-p_{i}$ on vertex $i$. The fiber $\psi^{-1}(q) \cap \mathcal{F}_{G}(\mathbf{a})$ of any point $q \in \mathcal{F}_{G}\left(a_{1}-p_{1}, \ldots, a_{n}-p_{n}, 0\right)$ is equal to $\{q\} \times \prod_{i \in T_{G}} p_{i} \Delta_{I_{i}}$. The claim follows. 
Corollary 4.3. We have

$$
\sigma_{G(\mathbf{a})}^{\varphi}\left(x_{i ; k}\right)=\sum_{\mathbf{p} \in \mathcal{P}(G ; \mathbf{a}) \cap \mathbb{Z}^{S_{G}}} K_{G}\left(a_{1}-\operatorname{ef}(\mathbf{p})_{1}, \ldots, a_{n}-\operatorname{ef}(\mathbf{p})_{n}, 0\right) \mathbf{x}^{\mathbf{p}},
$$

and

$$
\sigma_{G(\mathbf{a})}^{\psi}\left(x_{i}\right)=\sum_{\mathbf{p} \in \mathcal{Q}(G ; \mathbf{a}) \cap \mathbb{Z}^{T} G} K_{G}\left(a_{1}-p_{1}, \ldots, a_{n}-p_{n}, 0\right)\left(\begin{array}{c}
\left|I_{1}\right|+p_{1}-1 \\
\left|I_{1}\right|-1
\end{array}\right) \ldots\left(\begin{array}{c}
\left|I_{n}\right|+p_{n}-1 \\
\left|I_{n}\right|-1
\end{array}\right) \mathbf{x}^{\mathbf{p}} .
$$

Proof. The number of integer points of $\mathcal{F}_{G}(\mathbf{a})$ is given by the Kostant partition function $K_{G}(\mathbf{a})$. Combining this fact with Theorem 4.1 and Corollary 4.2 gives the desired result.

\section{Normalized PRoJeCtions of INTEGER POINT TRANSForms ARE LORENTZIAN}

In this section, we show that $N\left(\sigma_{G(\mathbf{a})}^{\varphi}\right)$ and $N\left(\sigma_{G(\mathbf{a})}^{\psi}\right)$ are Lorentzian; see Theorem 5.1. In order to prove this we begin with a series of reductions (Proposition 5.8 and Lemma 5.11). Then, a combinatorial symmetry (Lemma 5.14) allows us to realize Hessians of repeated partial derivatives of $\sigma_{G(\mathbf{a})}^{\varphi}$ as Hessians of repeated partial derivatives of volume polynomials.

We begin by formally stating the main result of this section.

THEOREM 5.1. The polynomials $\left(N\left(\sigma_{G(\mathbf{a})}^{\varphi}\right)\right)\left(x_{i ; k}\right)$ and $\left(N\left(\sigma_{G(\mathbf{a})}^{\psi}\right)\right)\left(x_{i}\right)$ are Lorentzian. Corollary 5.2 (cf. [5, Proposition 11]). For any directed graph $G$ on the vertex set $[n]$ and for any $\mathbf{v} \in \mathbb{Z}^{n}$ we have:

$$
K_{G}(\mathbf{v})^{2} \geqslant K_{G}\left(\mathbf{v}+e_{i}-e_{j}\right) K_{G}\left(\mathbf{v}-e_{i}+e_{j}\right)
$$

for every $i, j \in[n]$.

Proof. As $N\left(\sigma_{G(\mathbf{a})}^{\varphi}\right)$ is Lorentzian, the coefficients of $\sigma_{G(\mathbf{a})}^{\varphi}$ satisfy a log-concavity inequality (see Proposition 2.3). This inequality is precisely Equation (10).

Note that Corollary 5.2 also follows from the classical Alexandrov-Fenchel inequalities for mixed volumes, since $K_{G}(\mathbf{v})$ can be seen as mixed volumes of Minkowski sums of flow polytopes.

A first stepping stone towards Theorem 5.1 is to reduce to the problem of showing $N\left(\sigma_{G(\mathbf{a})}^{\varphi}\right)$ is Lorentzian for all $G$; this is the content of Proposition 5.8. In order to do this we introduce the following construction.

Definition 5.3. For a graph $G$, we denote by $G^{\mathrm{ex}}=\left(V^{\mathrm{ex}}, E^{\mathrm{ex}}\right)$ the graph obtained from $G$ by adding formal vertices $i^{\mathrm{ex}}$ for each vertex $i \in T_{G}$, by replacing edges $(i, n+1 ; j) \in S_{G}$ with edges $\left(i, i^{\mathrm{ex}} ; j\right)$, and by adding edges $\left(i^{\mathrm{ex}}, n+1 ; 1\right)$ for each $i^{\text {ex }} \in T_{G}$. Formally, we have

$$
\begin{aligned}
& V^{\text {ex }} \stackrel{\text { def }}{=}[n] \sqcup\left\{i^{\text {ex }}: i \in T_{G}\right\} \sqcup\{n+1\}, \\
& E^{\text {ex }} \stackrel{\text { def }}{=}\left(E \backslash S_{G}\right) \sqcup\left\{\left(i, i^{\text {ex }} ; j\right):(i, n+1 ; j) \in S_{G}\right\} \sqcup\left\{\left(i^{\text {ex }}, n+1 ; 1\right): i \in T_{G}\right\} .
\end{aligned}
$$

See Figure 2 for an example. The graph $G$ can be recovered from $G^{\mathrm{ex}}$ by a series of contractions, so we call $G^{\text {ex }}$ the extension of $G$.

Definition 5.4. For any two vectors $\mathbf{p}=\left(p_{1}, \ldots, p_{m}\right) \in \mathbb{R}^{m}$ and $\mathbf{q}=\left(q_{1}, \ldots, q_{n}\right) \in$ $\mathbb{R}^{n}$, we denote by $\mathbf{p} \oplus \mathbf{q} \in \mathbb{R}^{m+n}$ their concatenation, that is,

$$
\mathbf{p} \oplus \mathbf{q}=\left(p_{1}, \ldots, p_{m}, q_{1}, \ldots, q_{n}\right) \in \mathbb{R}^{m+n} .
$$



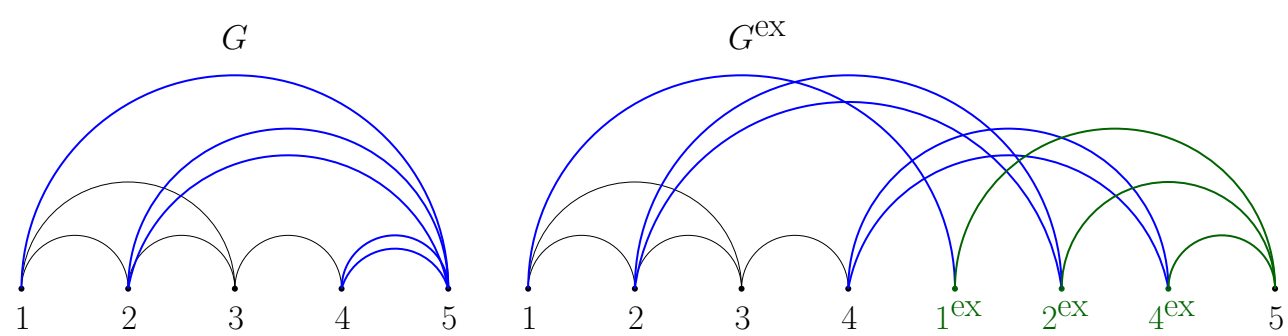

Figure 2. The graph $G$ in Figure 1, along with $G^{\text {ex }}$, defined in Definition 5.3.

For a netflow a for $G$ satisfying the conventions of this paper, we denote by $\mathbf{a}^{\mathrm{ex}}$ the netflow for $G^{\mathrm{ex}}$ given by

$$
\left.\mathbf{a}\right|_{[n]} \oplus \mathbf{0}_{T_{G} \mathrm{ex}} \oplus-a_{n+1}=(a_{1}, \ldots, a_{n}, \underbrace{0, \ldots, 0}_{\mid T_{G} \text { ex } \mid \text { many }},-a_{n+1}) ;
$$

note that $\mathbf{a}^{\mathrm{ex}}$ also satisfies the conventions of this paper.

Lemma 5.5. The bijection $T_{G} \leftrightarrow S_{G^{\mathrm{ex}}}$ given by $i \leftrightarrow\left(i^{\mathrm{ex}}, n+1 ; 1\right)$ induces an isomorphism on the real vector spaces $\mathbb{R}^{T_{G}}$ and $\mathbb{R}^{S_{G}{ }^{\mathrm{ex}}}$ by renaming basis elements according to the bijection. This isomorphism restricts to an integral equivalence $\mathcal{Q}(G ; \mathbf{a}) \equiv$ $\mathcal{P}\left(G^{\mathrm{ex}} ; \mathbf{a}^{\mathrm{ex}}\right)$.

Proof. By definition,

$$
\mathcal{Q}(G ; \mathbf{a})=\sum_{i \in[n]} a_{i} \Delta_{T_{G, i}} \quad \text { and } \quad \mathcal{P}\left(G^{\mathrm{ex}} ; \mathbf{a}^{\mathrm{ex}}\right)=\sum_{i \in[n] \sqcup T_{G}^{\mathrm{ex}}} a_{i}^{\mathrm{ex}} \Delta_{S_{G}{ }^{\mathrm{ex}, i}} .
$$

Since for every $i \in T_{G^{\text {ex }}}$ we have $a_{i}^{\text {ex }}=0$, and for every $i \in[n]$ we have $a_{i}^{\text {ex }}=a_{i}$, we may write

$$
\mathcal{P}\left(G^{\mathrm{ex}} ; \mathbf{a}^{\mathrm{ex}}\right)=\sum_{i \in[n]} a_{i} \Delta_{S_{G}^{\mathrm{ex}}, i}
$$

furthermore, we have $S_{G^{\mathrm{ex}}, i}=\left\{\left(j^{\mathrm{ex}}, n+1 ; 1\right): j \in T_{G, i}\right\}$. Thus, the isomorphism sends $\Delta_{T_{G, i}}$ to $\Delta_{S_{G} \text { ex }, i}$; passing to the Minkowski sum, we obtain the integral equivalence $\mathcal{Q}(G ; \mathbf{a}) \equiv \mathcal{P}\left(G^{\mathrm{ex}} ; \mathbf{a}^{\mathrm{ex}}\right)$.

LEMma 5.6. The bijection $E(G) \leftrightarrow E\left(G^{\mathrm{ex}}\right) \backslash S_{G^{\mathrm{ex}}}$ given by sending an edge $(i, n+$ $1 ; k) \in S_{G}$ to $\left(i, i^{\mathrm{ex}} ; k\right) \in E\left(G^{\mathrm{ex}}\right) \backslash S_{G^{\mathrm{ex}}}$ and an edge $(i, j ; k) \in E(G) \backslash S_{G}$ to $(i, j ; k) \in$ $E\left(G^{\mathrm{ex}}\right) \backslash S_{G^{\mathrm{ex}}}$ induces an isomorphism on the real vector spaces spanned by $E(G)$ and $E\left(G^{\mathrm{ex}}\right) \backslash S_{G^{\mathrm{ex}}}$ by renaming basis elements according to the bijection. For every $\mathbf{q} \in \mathcal{Q}(G ; \mathbf{a})$, this isomorphism restricts to an integral equivalence

$$
\mathcal{F}_{\left.G\right|_{[n]}}\left(\left.\mathbf{a}\right|_{[n]}-\mathbf{q}\right) \times \prod_{i \in T_{G}} q_{i} \Delta_{I_{i}} \equiv \mathcal{F}_{\left.G^{\mathrm{ex}}\right|_{[n] \sqcup T_{G} \mathrm{ex}}}\left(\left.\mathbf{a}\right|_{[n]} \oplus(-\mathbf{q})\right) .
$$

In light of Corollary 4.2, note that the left side of Equation (11) is the fiber of $\mathbf{q}$ under $\mathcal{F}_{G}(\mathbf{a}) \rightarrow \mathcal{Q}(G ; \mathbf{a})$. For brevity of notation, let us temporarily denote by $\widetilde{\mathbf{q}} \in$ $\mathbb{Z}^{S_{G} \text { ex }}$ the image of $\mathbf{q} \in \mathbb{Z}^{T_{G}}$ under the isomorphism in Lemma 5.5. In this notation, Theorem 4.1 implies that the right side of Equation (11) is (integrally equivalent to) the fiber of $\widetilde{\mathbf{q}} \in \mathbb{Z}^{S_{G^{\mathrm{ex}}}}$ under $\mathcal{F}_{G^{\mathrm{ex}}}\left(\mathbf{a}^{\mathrm{ex}}\right) \rightarrow \mathcal{P}\left(G^{\mathrm{ex}} ; \mathbf{a}^{\mathrm{ex}}\right)$.

We emphasize here that there is an integral equivalence $\mathcal{F}_{\left.G\right|_{[n]}}\left(\left.\mathbf{a}\right|_{[n]}-\mathbf{q}\right) \equiv$ $\mathcal{F}_{G}\left(\left.\mathbf{a}\right|_{[n]}-\mathbf{q}, 0\right)$.

Proof of Lemma 5.6. A point $f \in \mathcal{F}_{\left.G\right|_{[n]}}\left(\left.\mathbf{a}\right|_{[n]}-\mathbf{q}\right) \times \prod_{i \in T_{G}} q_{i} \Delta_{I_{i}}$ can be interpreted as a flow in $\mathcal{F}_{G}(\mathbf{a})$ with outflow $q_{i}$ at each vertex $i \in T_{G}$, by Corollary 4.2. Under the 
isomorphism in Lemma 5.6, $f$ gets mapped to a flow in $\left.G^{\mathrm{ex}}\right|_{[n] \sqcup T_{\mathrm{G}} \text { ex }}$ with netflow $a_{i}$ at each vertex $i \in[n]$ and netflow $-q_{i}$ at each vertex $i^{\text {ex }} \in T_{G^{\text {ex }}}$. In other words, the image is in $\left.\mathcal{F}_{G^{\mathrm{ex}}}\right|_{[n] \sqcup T_{G^{\mathrm{ex}}}}\left(\left.\mathbf{a}\right|_{[n]} \oplus(-\mathbf{q})\right)$.

Conversely, the preimage of a flow $\mathcal{F}_{\left.G^{\mathrm{ex}}\right|_{[n] \sqcup T_{G} \text { ex }}}\left(\left.\mathbf{a}\right|_{[n]} \oplus(-\mathbf{q})\right)$ is a flow in $\mathcal{F}_{G}(\mathbf{a})$ with netflow $q_{i}$ at each vertex $i \in T_{G}$; hence by Corollary 4.2 the preimage of $f$ is in $\mathcal{F}_{\left.G\right|_{[n]}}\left(\left.\mathbf{a}\right|_{[n]}-\mathbf{q}\right) \times \prod_{i \in T_{G}} q_{i} \Delta_{I_{i}}$.

Lemma 5.7. The bijection $T_{G} \leftrightarrow S_{G^{\mathrm{ex}}}$ given by $i \leftrightarrow\left(i^{\mathrm{ex}}, n+1 ; 1\right)$ induces an isomorphism on the polynomial rings $\mathbb{R}\left[\left(x_{i}\right)_{i \in T_{G}}\right]$ and $\mathbb{R}\left[\left(x_{i}\right)_{i \in S_{G} \text { ex }}\right]$ by renaming variables according to the bijection. Under this isomorphism, the polynomial $N\left(\sigma_{G(\mathbf{a})}^{\psi}\right)$ is sent to $N\left(\sigma_{G^{\text {ex }}\left(\mathbf{a}^{\text {ex }}\right)}\right)$.

Proof. Explicitly, we need to show that the polynomials

$$
N\left(\sigma_{G(\mathbf{a})}^{\psi}\right)=\sum_{\mathbf{q} \in \mathcal{Q}(G ; \mathbf{a}) \cap \mathbb{Z}^{T} G}\left(\psi^{-1}(\mathbf{q}) \cap \mathbb{Z}^{E(G)}\right) \frac{\mathbf{x}^{\mathbf{q}}}{\mathbf{q} !}
$$

and

$$
N\left(\sigma_{G^{\text {ex }}\left(\mathbf{a}^{\text {ex }}\right)}^{\varphi}\right)=\sum_{\mathbf{p} \in \mathcal{P}\left(G^{\text {ex }}, \mathbf{a}^{\text {ex }}\right) \cap \mathbb{Z}^{S} G^{\text {ex }}}\left(\varphi^{-1}(\mathbf{p}) \cap \mathbb{Z}^{E\left(G^{\text {ex }}\right)}\right) \frac{\mathbf{x}^{\mathbf{p}}}{\mathbf{p} !}
$$

agree after renaming variables according to the bijection. We stress that the map $\psi$ in Equation (12) is the projection $\mathcal{F}_{G}(\mathbf{a}) \rightarrow \mathcal{Q}(G ; \mathbf{a})$, whereas the map $\varphi$ in Equation (13) is the projection $\mathcal{F}_{G^{\mathrm{ex}}}\left(\mathbf{a}^{\mathrm{ex}}\right) \rightarrow \mathcal{P}\left(G^{\mathrm{ex}} ; \mathbf{a}^{\mathrm{ex}}\right)$.

By Lemma 5.5, the monomials $\mathrm{x}^{\mathbf{q}}$ appearing in Equation (12) and the monomials $\mathbf{x}^{\mathbf{p}}$ appearing in Equation (13) correspond to each other under the isomorphism $\mathbb{R}\left[\left(x_{i}\right)_{i \in T_{G}}\right] \cong \mathbb{R}\left[\left(x_{i}\right)_{i \in S_{G} \text { ex }}\right]$.

By Lemma 5.6, the fibers $\psi^{-1}(\mathbf{q})$ appearing in Equation (12) and the corresponding fibers $\varphi^{-1}(\mathbf{p})$ appearing in Equation (13) are integrally equivalent. Hence the coefficients of the monomials appearing in Equations (12) and (13) match.

Proposition 5.8. Suppose $N\left(\sigma_{G(\mathbf{a})}^{\varphi}\right)$ is Lorentzian for every $G$. Then $N\left(\sigma_{G(\mathbf{a})}^{\psi}\right)$ is Lorentzian for every $G$.

Proof. Lemma 5.7 asserts that up to renaming variables, we have the equality

$$
N\left(\sigma_{G(\mathbf{a})}^{\psi}\right)=N\left(\sigma_{G^{\text {ex }}\left(\mathbf{a}^{\mathrm{ex}}\right)}^{\varphi}\right) .
$$

By assumption, $N\left(\sigma_{G^{\text {ex }}\left(\mathbf{a}^{\text {ex }}\right)}\right)$ is Lorentzian.

LEMmA 5.9. Let $\mathbf{d} \in\left(\sum_{i=1}^{n} a_{i}-2\right) \Delta_{S_{G}} \cap \mathbb{Z}^{S_{G}}$ be an integer point in the scaled coordinate simplex of $S_{G}$. Suppose that the $\left|S_{G}\right| \times\left|S_{G}\right|$ matrix

$$
K_{\mathbf{d}} \stackrel{\text { def }}{=}\left(k_{i, j}\right)_{i, j \in S_{G}} ; \quad k_{\left(i_{1} ; k_{1}\right),\left(i_{2} ; k_{2}\right)} \stackrel{\text { def }}{=} K_{\left.G\right|_{[n]}}\left(\left.\mathbf{a}\right|_{[n]}-\operatorname{ef}(\mathbf{d})-e_{i_{1}}-e_{i_{2}}\right)
$$

has at most one positive eigenvalue. Then $N\left(\sigma_{G(\mathbf{a})}^{\varphi}\right)$ is Lorentzian.

Proof. The support of $N\left(\sigma_{G(\mathbf{a})}^{\varphi}\right)$ is M-convex by Proposition 3.4.

By Corollary 4.3, the $i j$-th element of $K_{\mathbf{d}}$ is the coefficient of $\mathbf{x}^{\mathbf{d}+e_{i}+e_{j}}$ in $\sigma_{G(\mathbf{a})}^{\varphi}$; equivalently, $K_{\mathbf{d}}$ is the Hessian of $\partial_{\mathbf{d}} N\left(\sigma_{G(\mathbf{a})}^{\varphi}\right)$. Since, by assumption, $K_{\mathbf{d}}$ has at most one positive eigenvalue, Lemma 2.2 asserts that $N\left(\sigma_{G(\mathbf{a})}^{\varphi}\right)$ is Lorentzian.

DeFinition 5.10. For a graph $G$ as in the conventions of this section, denote by $G^{-}$ the graph with vertex set $[n+1]$ and edge set

$$
E\left(G^{-}\right)=E\left(\left.G\right|_{[n]}\right) \sqcup\left\{e \in S_{G}: e=(i, n+1 ; 1)\right\} .
$$


In other words, $G^{-}$is obtained from $G$ by replacing, for each $i \in[n]$ with $M(i, n+1) \geqslant$ 1 , the set of edges connecting $i$ to the sink with a single edge connecting $i$ to the sink. See Figure 3 for an example. Note that since $G^{-}$has at most one edge connecting $i$ to $n+1$ for any $i$, we have $S_{G^{-}}=T_{G^{-}}=T_{G}$; we index the variables appearing in $\sigma_{G^{-}(\mathbf{a})}^{\varphi}$ with $\left(x_{i}\right)_{i \in T_{G}}$.
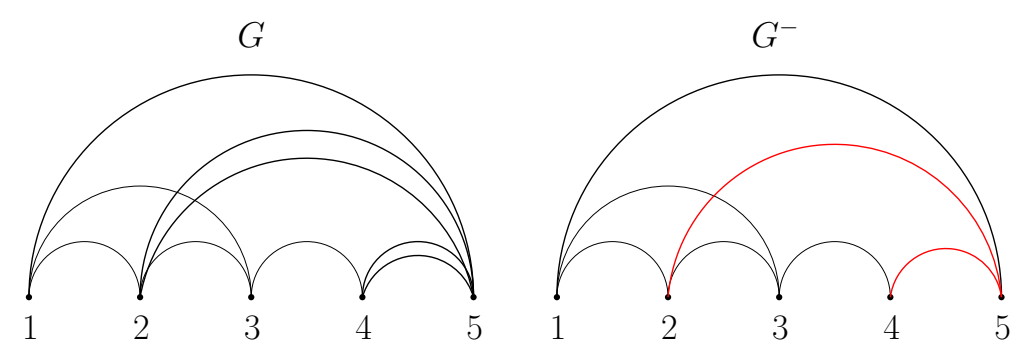

Figure 3. The graph $G$ from Figures 1 and 2. The graph $G^{-}$constructed from $G$ is shown beside it; see Definition 5.10 .

LEMma 5.11. Suppose $\left(N\left(\sigma_{G^{-}(\mathbf{a})}^{\varphi}\right)\right)\left(x_{i}\right)$ is Lorentzian. Then $\left(N\left(\sigma_{G(\mathbf{a})}^{\varphi}\right)\right)\left(x_{i ; k}\right)$ is also Lorentzian.

Proof. The support of $N\left(\sigma_{G(\mathbf{a})}^{\varphi}\right)$ is M-convex by Proposition 3.4.

By Lemma 5.9, we need to show that for every $\mathbf{d} \in\left(\sum_{i=1}^{n} a_{i}-2\right) \Delta_{S_{G}} \cap \mathbb{Z}^{S_{G}}$, the $\left|S_{G}\right| \times\left|S_{G}\right|$ matrix

$$
K_{\mathbf{d}}=\left(k_{i, j}\right)_{i, j \in S_{G}} ; \quad k_{\left(i_{1} ; k_{1}\right),\left(i_{2} ; k_{2}\right)}=K_{\left.G\right|_{[n]}}\left(\left.\mathbf{a}\right|_{[n]}-\mathrm{ef}(\mathbf{d})-e_{i_{1}}-e_{i_{2}}\right)
$$

has at most one positive eigenvalue. The matrix $K_{\mathbf{d}}$ is obtained from the $\left|T_{G}\right| \times\left|T_{G}\right|$ matrix

$$
K_{\mathrm{ef}(\mathbf{d})}^{-} \stackrel{\text { def }}{=}\left(k_{i, j}^{-}\right)_{i, j \in T_{G}} ; \quad k_{i, j}^{-} \stackrel{\text { def }}{=} K_{\left.G\right|_{[n]}}\left(\left.\mathbf{a}\right|_{[n]}-\mathrm{ef}(\mathbf{d})-e_{i}-e_{j}\right)
$$

first by repeating the $i$-th row $M(i, n+1)$ many times for each $i$, and then by repeating the $i$-th column $M(i, n+1)$ many times for each $i$. Note that the rank of $K_{\mathrm{ef}(\mathrm{d})}^{-}$is equal to the rank of $K_{\mathbf{d}}$; we write

$$
r \stackrel{\text { def }}{=} \operatorname{rank}\left(K_{\mathrm{ef}(\mathbf{d})}^{-}\right)=\operatorname{rank}\left(K_{\mathbf{d}}\right) .
$$

Observe, by Corollary 4.3 that the $i j$-th entry of $K_{\mathrm{ef}(\mathbf{d})}^{-}$is the coefficient of $\mathbf{x}^{\text {ef }(\mathbf{d})+e_{i}+e_{j}}$ in $\sigma_{G^{-}(\mathbf{a})}^{\varphi}$. By assumption, $N\left(\sigma_{G^{-}(\mathbf{a})}^{\varphi}\right)$ is Lorentzian; hence, Lemma 2.2 asserts that $K_{\mathrm{ef}(\mathbf{d})}^{-}$has at most one positive eigenvalue for any ef $(\mathbf{d}) \in\left(\sum_{i=1}^{n} a_{i}\right.$ 2) $\Delta_{T_{G}} \cap \mathbb{Z}^{T_{G}}$. In particular it has at least $r-1$ negative eigenvalues. Note also that $K_{\text {ef(d) }}^{-}$is a principal submatrix of $K_{\mathbf{d}}$; by Cauchy's Interlacing Theorem (Proposition 2.4), the eigenvalues $\alpha_{1} \leqslant \alpha_{2} \leqslant \cdots \leqslant \alpha_{\left|S_{G}\right|}$ of $K_{\mathbf{d}}$ and the eigenvalues $\beta_{1} \leqslant \cdots \leqslant \beta_{\left|T_{G}\right|}$ of $K_{\text {ef }(\mathbf{d})}^{-}$satisfy

$$
\alpha_{i} \leqslant \beta_{i} \quad \text { for all } 1 \leqslant i \leqslant\left|T_{G}\right| \text {. }
$$

Since $K_{\mathrm{ef}(\mathbf{d})}^{-}$has at least $r-1$ negative eigenvalues,

$$
\alpha_{i} \leqslant \beta_{i}<0 \quad \text { for all } 1 \leqslant i \leqslant r-1,
$$

so $K_{\mathbf{d}}$ also has at least $r-1$ negative eigenvalues. Furthermore, $K_{\mathbf{d}}$ has rank $r$. Hence, $K_{\mathbf{d}}$ also has at most one positive eigenvalue, and $\left(N\left(\sigma_{G(\mathbf{a})}^{\varphi}\right)\right)\left(x_{i ; k}\right)$ is Lorentzian. 
ExAMPLE 5.12. Let $G$ be as in Figure 3 and $\mathbf{a}=(2,1,1,1,-5)$. Let $\mathbf{d} \in 3 \Delta_{S_{G}} \cap \mathbb{Z}^{S_{G}}$ be the vector $e_{2 ; 1}+e_{2 ; 2}+e_{4 ; 2}$; this integer vector takes the value 1 on the edges $(2,5 ; 1),(2,5 ; 2),(4,5 ; 2) \in S_{G}$ and takes the value 0 everywhere else. Thus $\left.\mathbf{a}\right|_{[n]}-$ ef $(\mathbf{d})=(2,-1,1,0)$. The matrix $K_{\mathbf{d}}$ is given by

$$
\left[\begin{array}{lllll}
k_{(1 ; 1),(1 ; 1)} & k_{(1 ; 1),(2 ; 1)} & k_{(1 ; 1),(2 ; 2)} & k_{(1 ; 1),(4 ; 1)} & k_{(1 ; 1),(4 ; 2)} \\
k_{(2 ; 1),(1 ; 1)} & k_{(2 ; 1),(2 ; 1)} & k_{(2 ; 1),(2 ; 2)} & k_{(2 ; 1),(4 ; 1)} & k_{(2 ; 1),(4 ; 2)} \\
k_{(2 ; 2),(1 ; 1)} & k_{(2 ; 2),(2 ; 1)} & k_{(2 ; 2),(2 ; 2)} & k_{(2 ; 2),(4 ; 1)} & k_{(2 ; 2),(4 ; 2)} \\
k_{(4 ; 1),(1 ; 1)} & k_{(4 ; 1),(2 ; 1)} & k_{(4 ; 1),(2 ; 2)} & k_{(4 ; 1),(4 ; 1)} & k_{(4 ; 1),(4 ; 2)} \\
k_{(4 ; 2),(1 ; 1)} & k_{(4 ; 2),(2 ; 1)} & k_{(4 ; 2),(2 ; 2)} & k_{(4 ; 2),(4 ; 1)} & k_{(4 ; 2),(4 ; 2)}
\end{array}\right]=\left[\begin{array}{lllll}
0 & 0 & 0 & 1 & 1 \\
0 & 0 & 0 & 1 & 1 \\
0 & 0 & 0 & 1 & 1 \\
1 & 1 & 1 & 2 & 2 \\
1 & 1 & 1 & 2 & 2
\end{array}\right] .
$$

It is obtained from the matrix $K_{\mathrm{ef}(\mathbf{d})}^{-}$given by

$$
\left[\begin{array}{lll}
k_{1,1}^{-} & k_{1,2}^{-} & k_{1,4}^{-} \\
k_{2,1}^{-} & k_{2,2}^{-} & k_{2,4}^{-} \\
k_{4,1}^{-} & k_{4,2}^{-} & k_{4,4}^{-}
\end{array}\right]=\left[\begin{array}{lll}
0 & 0 & 1 \\
0 & 0 & 1 \\
1 & 1 & 2
\end{array}\right]
$$

first by repeating the second row $M(2,5)=2$ times and repeating the third row $M(4,5)=2$ times, to obtain

$$
\left[\begin{array}{lll}
0 & 0 & 1 \\
0 & 0 & 1 \\
0 & 0 & 1 \\
1 & 1 & 2 \\
1 & 1 & 2
\end{array}\right]
$$

and then repeating the second column $M(2,5)=2$ times and repeating the third column $M(4,5)=2$ times, to obtain

$$
\left[\begin{array}{lllll}
0 & 0 & 0 & 1 & 1 \\
0 & 0 & 0 & 1 & 1 \\
0 & 0 & 0 & 1 & 1 \\
1 & 1 & 1 & 2 & 2 \\
1 & 1 & 1 & 2 & 2
\end{array}\right]
$$

The spectrum for $K_{\text {ef }(\mathbf{d})}^{-}$is $\{-\sqrt{3}+1,0, \sqrt{3}+1\}$ (which has at most one positive eigenvalue).

In this example, the ranks of $K_{\mathrm{ef}(\mathbf{d})}^{-}$and $K_{\mathbf{d}}$ are both equal to 2 , thus they both have a total of 2 nonzero eigenvalues. The matrix $K_{\text {ef (d) }}^{-}$is the principal submatrix of $K_{\mathbf{d}}$ corresponding to the 1 st, 2 nd, and 4 th rows and columns of $K_{\mathbf{d}}$. Cauchy's Interlacing Theorem says that the smallest eigenvalue of $K_{\mathbf{d}}$ is at most $-\sqrt{3}+1<0$. Hence $K_{\mathbf{d}}$ has at most one positive eigenvalue.

DEFINITION 5.13. For a graph $G$ as in the conventions of this section, denote by $G^{r}$ the graph obtained by "flipping" $\left.G\right|_{[n]}$, that is, $V\left(G^{r}\right)=[n]$ and

$$
(i, j) \in E\left(G^{r}\right) \Longleftrightarrow(n+1-j, n+1-i) \in E\left(\left.G\right|_{[n]}\right) .
$$

Equivalently, $G^{r}$ is obtained by relabeling the vertices of $\left.G\right|_{[n]}$ by the map $i \mapsto n+1-i$ and reversing the orientation of edges. See Figure 4 for an example.

The symmetry between $\left.G\right|_{[n]}$ and $G^{r}$ underpins the following lemma, crucial for the proof of Theorem 5.1:

LEMma 5.14 ([6, Corollary 2.4]). For every $c_{1}, \ldots, c_{n} \in \mathbb{Z}$, the formula

$$
K_{\left.G\right|_{[n]}}\left(c_{1}, c_{2}, \ldots, c_{n-1}, c_{n}\right)=K_{G^{r}}\left(-c_{n},-c_{n-1}, \ldots,-c_{2},-c_{1}\right)
$$

holds. 

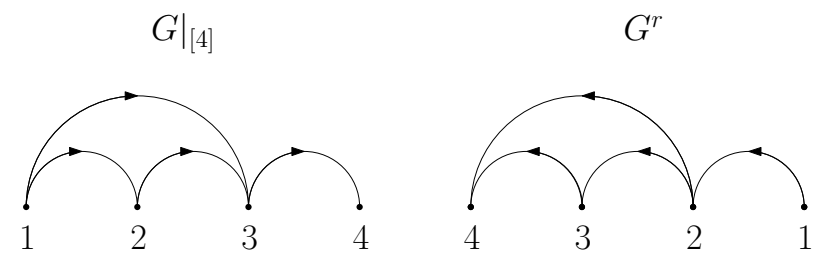

FiguRE 4. The graphs $\left.G\right|_{[4]}$ and $G^{r}$ are shown, for $G$ as in Figure 3 and Example 5.12.

Definition 5.15. Denote by $P_{T}$ the permutation matrix corresponding to the orderreversing permutation $i \mapsto\left|T_{G}\right|+1-i$; this is the matrix consisting of 1 's on the antidiagonal and 0 everywhere else.

Observe that

$$
\widetilde{K}_{\mathrm{ef}(\mathbf{d})}^{-} \stackrel{\text { def }}{=} P_{T} K_{\mathrm{ef}(\mathbf{d})}^{-} P_{T}
$$

has the same spectrum as $K_{\mathrm{ef}(\mathbf{d})}^{-}$since it is obtained by conjugation. We index the rows and columns of $\widetilde{K}_{\mathrm{ef}(\mathbf{d})}^{-}$by $\left\{i: n+1-i \in T_{G}\right\}$ (in increasing order). See Example 5.16.

ExAmPle 5.16. Let $G$ be as in Figure 3, Example 5.12, and Figure 4. The entries of $K_{\text {ef(d) }}$ - are given by

$$
\left[\begin{array}{lll}
k_{1,1}^{-} & k_{1,2}^{-} & k_{1,4}^{-} \\
k_{2,1}^{-} & k_{2,2}^{-} & k_{2,4}^{-} \\
k_{4,1}^{-} & k_{4,2}^{-} & k_{4,4}^{-}
\end{array}\right]=\left[\begin{array}{lll}
0 & 0 & 1 \\
0 & 0 & 1 \\
1 & 1 & 2
\end{array}\right] .
$$

Then the matrix $\widetilde{K}_{\mathrm{ef}(\mathbf{d})}^{-}=P_{T} K_{\mathrm{ef}(\mathbf{d})}^{-} P_{T}$ is given by

$$
\left[\begin{array}{lll}
\widetilde{k}_{1,1}^{-} & \widetilde{k}_{1,3}^{-} & \widetilde{k}_{1,4}^{-} \\
\widetilde{k}_{3,1}^{-} & \widetilde{k}_{3,3}^{-} & \widetilde{k}_{3,4}^{-} \\
\widetilde{k}_{4,1}^{-} & \widetilde{k}_{4,3}^{-} & \widetilde{k}_{4,4}^{-}
\end{array}\right]=\left[\begin{array}{lll}
0 & 0 & 1 \\
0 & 1 & 0 \\
1 & 0 & 0
\end{array}\right]\left[\begin{array}{lll}
0 & 0 & 1 \\
0 & 0 & 1 \\
1 & 1 & 2
\end{array}\right]\left[\begin{array}{lll}
0 & 0 & 1 \\
0 & 1 & 0 \\
1 & 0 & 0
\end{array}\right]=\left[\begin{array}{lll}
2 & 1 & 1 \\
1 & 0 & 0 \\
1 & 0 & 0
\end{array}\right] \text {. }
$$

Proposition 5.17. The entries of $\widetilde{K}_{\mathrm{ef}(\mathbf{d})}^{-}$are given by the following formula. Let

$$
\mathbf{z}=\left(\operatorname{ef}(\mathbf{d})_{n}-a_{n}, \operatorname{ef}(\mathbf{d})_{n-1}-a_{n-1}, \ldots, \text { ef }(\mathbf{d})_{2}-a_{2}, \text { ef }(\mathbf{d})_{1}-a_{1}\right)
$$

Then the $i, j$-th entry $\widetilde{k}_{i, j}^{-}$of $\widetilde{K}_{\mathrm{ef}(\mathbf{d})}^{-}$is $K_{G^{r}}\left(\mathbf{z}+e_{i}+e_{j}\right)$.

Proof. The $i, j$-th entry $\tilde{k}_{i, j}^{-}$of $\widetilde{K}_{\mathrm{ef}(\mathbf{d})}^{-}$is the $(n+1-i, n+1-j)$-th entry of $K_{\mathrm{ef}(\mathbf{d})}^{-}$. This is equal to

$$
k_{n+1-i, n+1-j}^{-}=K_{\left.G\right|_{[n]}}\left(\left.\mathbf{a}\right|_{[n]}-\operatorname{ef}(\mathbf{d})-e_{n+1-i}-e_{n+1-j}\right)=K_{G^{r}}\left(\mathbf{z}+e_{i}+e_{j}\right),
$$

where the last equality is an application of Lemma 5.14 .

The final piece required to prove Theorem 5.1 is the existence of a quadratic Lorentzian polynomial whose Hessian is $\widetilde{K}_{\mathrm{ef}(\mathbf{d})}^{-}$. We are ready to accomplish this now:

Proof of Theorem 5.1. By Proposition 5.8, it suffices to show that $\left(N\left(\sigma_{G(\mathbf{a})}^{\varphi}\right)\right)\left(x_{i ; k}\right)$ is Lorentzian, and by Lemma 5.11, it suffices to show that $\left(N\left(\sigma_{G^{-}(\mathbf{a})}^{\varphi}\right)\right)\left(x_{i}\right)$ is Lorentzian. By Lemma 5.9 applied to $G^{-}$, we need to show that $K_{\mathbf{d}}=K_{\mathrm{ef}(\mathbf{d})}^{-}$has at most one positive eigenvalue for every $\mathbf{d} \in\left(\sum_{i=1}^{n} a_{i}-2\right) \Delta_{S_{G}} \cap \mathbb{Z}^{S_{G}}=\left(\sum_{i=1}^{n} a_{i}-2\right) \Delta_{T_{G}} \cap \mathbb{Z}^{T_{G}}$. In light of the discussion following Definition 5.15, it suffices to show, for every lattice point ef $(\mathbf{d}) \in\left(\sum a_{i}-2\right) \Delta_{T_{G}} \cap \mathbb{Z}^{T_{G}}$, that the matrix $\widetilde{K}_{\text {ef }(\mathbf{d})}^{-}$has at most one positive eigenvalue. 
For brevity of notation, we introduce

$$
\mathbf{z}=\left(z_{1}, \ldots, z_{n}\right)=\left(\operatorname{ef}(d)_{n}-a_{n}, \ldots, \operatorname{ef}(d)_{1}-a_{1}\right) ; \quad z_{\min } \stackrel{\text { def }}{=} \min _{i \in[n]} z_{i} .
$$

Note that $z_{\min }<0$, since $\sum z_{i}=-2$. Let $\widetilde{G}$ be the graph on the vertex set $\left[n+1-z_{\min }\right]$ with edges

$$
E(\widetilde{G})=E\left(G^{r}\right) \cup\{(i, j): i \leqslant j \text { and } n+1 \leqslant j\}
$$

Set

$$
\begin{array}{ll}
N \stackrel{\text { def }}{=} n-z_{\min } ; & \widetilde{\mathbf{z}} \stackrel{\text { def }}{=}(z_{1}, \ldots, z_{n}, \underbrace{0, \ldots, 0}_{-z_{\min }}) ; \\
\widetilde{\mathbf{x}} \stackrel{\text { def }}{=}\left(x_{1}, \ldots, x_{N+1}\right) ; & \widetilde{\mathbf{o}} \stackrel{\text { def }}{=}\left(\operatorname{outd}_{1}-1, \ldots, \text { outd }_{N}-1\right),
\end{array}
$$

where $\operatorname{outd}_{i}$ denotes the outdegree of $\widetilde{G}$ at vertex $i$. The Baldoni-Vergne formulas, Theorem 2.9 (see also Remark 2.10), applied to $\widetilde{G}$ says that

$$
\begin{aligned}
\operatorname{vol} \mathcal{F}_{\widetilde{G}}(\widetilde{\mathbf{x}}) & =\sum_{\substack{\mathbf{j}: \mathbf{j} \geqslant \mathbf{0} \\
j_{1}+\cdots+j_{N}=|E(\widetilde{G})|-N}} K_{\widetilde{G}}(\mathbf{j}-\widetilde{\mathbf{o}}) \frac{\left(\left.\widetilde{\mathbf{x}}\right|_{[N]}\right)^{\mathbf{j}}}{\mathbf{j} !} \\
& =\sum_{\substack{\mathbf{j}: \mathbf{j}+\widetilde{\mathbf{o}} \geqslant \mathbf{0} \\
j_{1}+\cdots+j_{N}=0}} K_{\widetilde{G}}(\mathbf{j}) \frac{\left(\left.\widetilde{\mathbf{x}}\right|_{[N]}\right)}{(\mathbf{j}+\widetilde{\mathbf{j}}+\widetilde{\mathbf{o}}) !}
\end{aligned}
$$

(Here, we stress that the $\mathbf{v} \geqslant \mathbf{w}$ means that $v_{i} \geqslant w_{i}$ for all $i$.) By Theorem 2.6, $\operatorname{vol} \mathcal{F}_{\widetilde{G}}(\widetilde{\mathbf{x}})$ is Lorentzian.

Note that $\widetilde{\mathbf{z}}+\widetilde{\mathbf{o}} \geqslant \mathbf{0}$, since for $i \leqslant n$ we have

$$
\widetilde{\mathbf{o}}_{i}=\operatorname{outd}_{i}-1 \geqslant|\{(i, j): n+1 \leqslant j \leqslant N+1\}|-1=N-n=-z_{\min },
$$

and for $i \geqslant n+1$ we have $\widetilde{\mathbf{z}}_{i}=0$. Thus, the partial derivative $\partial_{\widetilde{\mathbf{z}}+\widetilde{\mathbf{o}}}$ is well defined. We conclude that the polynomial

$$
\partial_{\widetilde{\mathbf{z}}+\widetilde{\mathbf{o}}} \operatorname{vol} \mathcal{F}_{\widetilde{G}}(\widetilde{\mathbf{x}})=\sum_{\substack{\mathbf{j}: \mathbf{j} \geqslant \widetilde{\mathbf{z}} \\ j_{1}+\cdots+j_{N}=0}} K_{\widetilde{G}}(\mathbf{j}) \frac{\left(\left.\widetilde{\mathbf{x}}\right|_{[N]}\right)^{\mathbf{j}-\widetilde{\mathbf{z}}}}{(\mathbf{j}-\widetilde{\mathbf{z}}) !}
$$

is Lorentzian. (The equality is an application of Lemma 2.1.)

Let $A$ be the $N \times N$ diagonal matrix whose $i$-th diagonal entry is 1 if $i \in\{n+1-$ $\left.j: j \in T_{G}\right\}$ and 0 otherwise; by Theorem 2.5 applied to $f=\partial_{\widetilde{\mathbf{z}}+\widetilde{\mathbf{o}}} \operatorname{vol} \mathcal{F}_{\widetilde{G}}(\widetilde{\mathbf{x}})$ and $A$ as above, the quadratic polynomial

$$
\partial_{\widetilde{\mathbf{z}}+\widetilde{\mathbf{o}}} \operatorname{vol} \mathcal{F}_{\widetilde{G}}(A \widetilde{\mathbf{x}})
$$

is Lorentzian and its Hessian has at most one positive eigenvalue. The rows and columns of this Hessian are naturally indexed by $\left\{n+1-j: j \in T_{G}\right\}$, and its $i, j$-th entry is the coefficient of $\frac{x_{i} x_{j}}{\left(e_{i}+e_{j}\right) !}$ in $\partial_{\widetilde{\mathbf{z}}+\widetilde{\mathbf{o}}} \operatorname{vol} \mathcal{F}_{\widetilde{G}}(A \widetilde{\mathbf{x}})$. This coefficient is $K_{G^{r}}\left(\widetilde{\mathbf{z}}+e_{i}+\right.$ $\left.e_{j}\right)$. By Proposition 5.17, its Hessian is precisely $\widetilde{K}_{\mathrm{ef}(\mathbf{d})}^{-}$.

We have thus shown that $\widetilde{K}_{\mathrm{ef}(\mathrm{d})}^{-}$has at most one positive eigenvalue, completing the proof. 


\section{ON PROJECTIONS OF POLYTOPES IN GENERAL}

Recall the question stemming from Theorem 5.1, as well as other examples mentioned in the Introduction:

QUESTION 1.1. Which polytope/projection pairs give rise to normalized projected integer point transforms that are Lorentzian?

Note that $\varphi$ is a projection onto a coordinate subspace and the flow polytope $\mathcal{F}_{G}(\mathbf{a})$ we are projecting lives in the nonnegative orthant. It is worth noting that if a Lorentzian polynomial $f$ equals the normalized projection onto a coordinate subspace of an integer point transform of a polytope which belongs to the nonnegative orthant, then any derivative of $f$ is (1) Lorentzian, (2) the normalized projection onto a coordinate subspace of an integer point transform of a polytope which belongs to the nonnegative orthant. We formalize this observation here.

Definition 6.1. A polytope/projection pair $(P, \varphi)$ is said to be admissible if the polytope $P \subseteq \mathbb{R}^{m}$ has vertices in $\mathbb{Z}^{m}$ and lives in the nonnegative orthant

$$
H_{+}^{m} \stackrel{\text { def }}{=}\left\{\left(x_{1}, \ldots, x_{m}\right): x_{i} \geqslant 0 \text { for all } i \in[m]\right\} ;
$$

we also require that $\varphi$ is a projection onto a coordinate $n$-dimensional subspace. Without loss of generality, we may assume $\varphi$ is projection onto the first $n$ components.

Observe that $\varphi(P) \subseteq H_{+}^{n}$ lives inside the nonnegative orthant of $\mathbb{R}^{n}$ and also has integral vertices.

To an admissible pair, we associate a polynomial $\sigma_{P}^{\varphi}$ obtained by projecting the integer point transform of $P$ according to $\varphi$; specifically,

$$
\sigma_{P}^{\varphi}(\mathbf{x}) \stackrel{\text { def }}{=} \sum_{\mathbf{p} \in P \cap \mathbb{Z}^{m}} \mathbf{x}^{\varphi(\mathbf{p})}=\sum_{\mathbf{p} \in \varphi(P) \cap \mathbb{Z}^{n}}\left(\varphi^{-1}(\mathbf{p}) \cap \mathbb{Z}^{m}\right) \mathbf{x}^{\mathbf{p}},
$$

where $\mathbf{x}=\left(x_{1}, \ldots, x_{n}\right)$, and $\varphi^{-1}(\mathbf{p})$ is interpreted as a subset of $P$. (Note that $\varphi(P) \subseteq H_{+}^{n}$ implies $\sigma_{P}^{\varphi}$ is actually a polynomial.)

Proposition 6.2. Let $f\left(x_{1}, \ldots, x_{n}\right)$ be a Lorentzian polynomial so that $f=N\left(\sigma_{P}^{\varphi}\right)$ for some admissible pair $(P, \varphi)$. Then we have

$$
\frac{\partial}{\partial x_{i}} f=N\left(\sigma_{P_{i}}^{\varphi}\right), \quad \text { where } \quad P_{i} \stackrel{\text { def }}{=}\left(P \cap H_{+i}^{m}\right)+\left\{-e_{i}\right\},
$$

where $H_{+i}^{m}=\left\{\left(x_{1}, \ldots, x_{m}\right): x_{i} \geqslant 1\right.$, and $x_{j} \geqslant 0$ for all $\left.j \in[m]\right\}$.

Proof. By Lemma 2.1, it suffices to show that

$$
\text { if } \quad \sigma_{P}^{\varphi}(\mathbf{x})=\sum_{\alpha} c_{\alpha} \mathbf{x}^{\alpha}, \quad \text { then } \quad \sigma_{P_{i}}^{\varphi}(\mathbf{x})=\sum_{\alpha: \alpha_{i} \geqslant 1} c_{\alpha} \mathbf{x}^{\alpha-e_{i}} .
$$

Since $e_{i} \in \mathbb{R}^{n}=\operatorname{im} \varphi$, we have $\varphi\left(P_{i}\right)=\varphi\left(P \cap H_{+i}^{m}\right)+\left\{-e_{i}\right\}=\varphi(P) \cap H_{+i}^{n}+\left\{-e_{i}\right\}$. A point $\beta \in \varphi\left(P_{i}\right)$ if and only if $\alpha \stackrel{\text { def }}{=} \beta+e_{i} \in \varphi(P) \cap H_{+i}^{n}$. Furthermore, the fiber $\varphi^{-1}(\beta) \cap P_{i}$ is equal, up to translation by $e_{i}$, to the fiber $\varphi^{-1}(\alpha) \cap P$. Thus

$$
\sigma_{P_{i}}^{\varphi}(\mathbf{x})=\sum_{\beta \in \varphi\left(P_{i}\right) \cap \mathbb{Z}^{n}}\left(\varphi^{-1}(\beta) \cap P_{i} \cap \mathbb{Z}^{n}\right) \mathbf{x}^{\beta}=\sum_{\substack{\alpha \in \varphi(P) \cap \mathbb{Z}^{n} \\ \alpha_{i} \geqslant 1}}\left(\varphi^{-1}(\alpha) \cap P \cap \mathbb{Z}^{n}\right) \mathbf{x}^{\alpha-e_{i}} .
$$

Comparing the above expression to the definition of $\sigma_{P}^{\varphi}$, we have verified Equation (14) holds. 
REMARK 6.3. We emphasize that the pair $\left(P_{i}, \varphi\right)$ is admissible when $(P, \varphi)$ is admissible. Furthermore, as discussed in the proof of Proposition 6.2,

$$
\varphi\left(P_{i}\right)=\varphi(P) \cap H_{+i}^{n}+\left\{-e_{i}\right\} .
$$

We conclude by another intriguing question stemming from our work: which Lorentzian polynomials arise naturally as normalized projections of integer point transforms of polytopes?

Acknowledgements. We are grateful to Ricky I. Liu as well as Dave Anderson and Eugene Gorsky for inspiring conversations related to the idea of polytope/projection pairs. We are also grateful to June Huh for inspiring conversations about Lorentzian polynomials as well as Louis Billera and Avery St. Dizier for inspiring conversations about polytopes in general and flow polytopes in particular. Finally, we would like to thank the referees for their careful reading and suggestions.

\section{REFERENCES}

[1] Spencer Backman, Christopher Eur, and Connor Simpson, Simplicial generation of Chow rings of matroids, https://arxiv.org/abs/1905.07114, 2019.

[2] Welleda Baldoni and Michèle Vergne, Kostant partitions functions and flow polytopes, Transform. Groups 13 (2008), no. 3-4, 447-469.

[3] Petter Brändén and June Huh, Lorentzian polynomials, Ann. of Math. (2) 192 (2020), no. 3, 821-891.

[4] Christopher Eur and June Huh, Logarithmic concavity for morphisms of matroids, Adv. Math. 367 (2020), Paper no. 107094 (19 pages).

[5] June Huh, Jacob P. Matherne, Karola Mészáros, and Avery St. Dizier, Logarithmic concavity of Schur and related polynomials, https://arxiv.org/abs/1906.09633, 2019.

[6] Karola Mészáros and Alejandro H. Morales, Volumes and Ehrhart polynomials of flow polytopes, Math. Z. 293 (2019), no. 3-4, 1369-1401.

[7] Karola Mészáros and Avery St. Dizier, From generalized permutahedra to Grothendieck polynomials via flow polytopes, Algebr. Comb. 3 (2020), no. 5, 1197-1229.

[8] Beresford N. Parlett, The symmetric eigenvalue problem, Classics in Applied Mathematics, vol. 20, Society for Industrial and Applied Mathematics (SIAM), Philadelphia, PA, 1998.

[9] Alexander Postnikov, Permutohedra, associahedra, and beyond, Int. Math. Res. Not. IMRN (2009), no. 6, 1026-1106.

[10] Alexander Schrijver, Combinatorial optimization. Polyhedra and efficiency. Vol. B, Algorithms and Combinatorics, vol. 24, Springer-Verlag, Berlin, 2003.

Karola MÉszÁros, Department of Mathematics, Cornell University, Ithaca, NY 14853, USA

E-mail : karola@math.cornell.edu

Linus Setiabrata, Department of Mathematics, University of Chicago, Chicago, IL 60637, USA

E-mail : linus@math.uchicago.edu 